\title{
Estado nutricional e percepção da imagem corporal de usuários da atenção básica de uma cidade do interior da Bahia
}

\section{Nutritional status and body image perception of primary care users in a city in the interior of} Bahia

DOI: $10.37111 /$ braspenj.2020352003

Ana Caroline de Sousa Almeida

Carolina Cunha de Oliveira ${ }^{2}$

Andrea Costa Goes ${ }^{3}$

Mylena Alves Santos ${ }^{3}$

Rafaela de Siqueira Oliveira ${ }^{3}$

\section{Unitermos:}

Aparência física. Imagem corporal. Estado nutricional. Adulto. Idoso.

\section{Keywords:}

Physical appearance, body. Body image. Nutritional status. Adult. Aged.

\section{Endereço de correspondência:}

Carolina Cunha de Oliveira.

Av. Governador Marcelo Deda, 13 - Centro - Lagarto,

SE, Brasil - CEP: 49400-000

E-mail: carol_cunh@yahoo.com.br

\section{Submissão}

18 de novembro de 2019

Aceito para publicação

20 de março de 2020

\begin{abstract}
RESUMO
Introdução: Atualmente, existe uma maior preocupação dos indivíduos com a visão e imposição de corpo perfeito. A alteração da percepção da imagem corporal é motivada por questões sociais, ambientais e, fortemente, pela mídia, a qual impõe um padrão de beleza associado à magreza ou aos corpos musculosos. Uma visão distorcida da imagem corporal real repercute nos níveis de bem-estar e autoestima, assim como nas tomadas de decisão relacionadas à nutrição e à saúde em geral. $O$ objetivo deste estudo é verificar o estado nutricional antropométrico e a percepção da imagem corporal de usuários da atenção básica. Método: Estudo transversal, conduzido com usuários da atenção básica, no município de Fátima-Bahia. Foram coletadas informações sociodemográficas, avaliação antropométrica (peso, altura e circunferência da cintura) e da percepção da imagem corporal ideal e real. Resultados: Participaram do estudo 89 usuários participantes do Programa HiperDia, sendo a maioria do sexo feminino $(87,6 \%)$ e adultos $(67,4 \%)$. Em relação ao estado nutricional, $61,8 \%$ apresentavam excesso de peso e 79,8\% possuíam acúmulo de gordura abdominal. A maioria dos indivíduos demonstrou insatisfação pelo excesso de peso $(78,7 \%)$ e $6,7 \%$ de insatisfação para magreza. Dentre os indivíduos com adequado estado nutricional, $73,5 \%$ também apresentavam algum tipo insatisfação corporal (seja pela magreza ou pelo excesso). Conclusão: A maioria dos indivíduos apresentava excesso de peso e insatisfação com a imagem corporal. É imprescindível uma avaliação da imagem corporal dos indivíduos por parte dos profissionais da saúde, auxiliando a prática clínica e garantindo uma atenção integral ao indivíduo.
\end{abstract}

\section{ABSTRACT}

Introduction: Currently, there is a greater concern of individuals with vision and imposition of perfect body. The change in the perception of the body image is motivated by social, environmental and, strongly, by the media, which imposes a beauty pattern associated with thinness or muscular bodies. A distorted view of the actual body image has repercussions on the levels of well-being and self-esteem, as well as in the decision-making related to nutrition and health in general. The objective of this study is to verify the anthropometric nutritional status and perception of the body image of users of primary care. Methods: A cross-sectional study conducted with users of primary care in the city of Fátima-Bahia. Socio-demographic information, anthropometric evaluation (weight, height and waist circumference) and perception of ideal and real body image were collected. Results: 89 users participating in the HiperDia program participated in the study, the most were women (87.6\%) and adults (67.4\%). Regarding nutritional status, 61.8\% were overweight and $79.8 \%$ had abdominal fat accumulation. Most of the individuals showed dissatisfaction due to overweight $(78.7 \%)$ and $6.7 \%$ of dissatisfaction for thinness. Of the individuals with adequate nutritional status, $73.5 \%$ also had some type of body dissatisfaction (both for thinness or excess). Conclusion: The majority of subjects presented with overweight and body image dissatisfaction. It is essential to evaluate the body image of the individuals on the part of the health professionals, assisting the clinical practice and guaranteeing an integral attention to the individual. 


\section{INTRODUÇ̃̃O}

$\mathrm{Na}$ atualidade, existe maior preocupação no que concerne à interpretação do corpo humano em sua plenitude, visto que a civilização vive em constante mudança na sua visão e imposição de corpo perfeito'.

A alteração na percepção da imagem corporal dos indivíduos é motivada por uma incessante procura por um padrão de beleza ideal, associada às realizações pessoais e à felicidade, especialmente no gênero feminino ${ }^{2}$. Enquanto antes a mulher perfeita tinha que ser magra e com corpo de modelo, atualmente surgem os corpos sarados e malhados como fundamental para estarem no universo da beleza, segundo as leis da mídia'.

O poder de persuasão da mídia é tão significativo que influencia tanto crianças e adolescentes, que estão aprendendo a lidar com as mudanças corporais durante seu desenvolvimento, quanto em adultos e idosos, os quais já poderiam estar mais seguros com o próprio corpo, evitando transtornos de imagem. Os idosos têm um agravante na percepção da imagem corporal por conta das modificações advindas da senescência, como alterações nos hábitos alimentares, sarcopenia, surgimento de doenças crônicas não transmissíveis (DCNT), solidão, uso de medicamentos que, somados à visão de corpo midiático, ocasionam distorções devido à ótica negativa no tangente à velhice ${ }^{1,3,4}$.

A autoimagem corporal é um fator que deve estar em consonância com as medidas antropométricas dos indivíduos. Porém, muitos têm uma visão distorcida do real, repercutindo negativamente nos níveis de bem-estar e autoestima, assim como nas tomadas de decisão relacionadas à nutrição e à saúde em geral ${ }^{3,5,6}$. Desta forma, torna-se importante conhecer a percepção da imagem corporal dos indivíduos, para que estes recebam orientações diante das percepções observadas, atendendo ao problema de forma especializada e eficaz. Assim, o presente estudo teve por objetivo verificar o estado nutricional antropométrico e a percepção da imagem corporal de usuários da atenção básica.

\section{MÉTODO}

Estudo transversal, quantitativo e de caráter descritivo, com coleta primária de dados, desenvolvido com usuários da atenção básica no município de Fátima, no interior da Bahia, participantes do Programa HiperDia do Núcleo de Apoio à Saúde da Família (NASF) do município.

A amostra foi composta por 89 usuários dos Programa HiperDia, de ambos os sexos, adultos e idosos. Para seleção dos usuários era necessária a participação nas atividades do HiperDia. Os usuários foram convocados nos dias de atividades do Programa ou por meio de visitas domiciliares em apoio com os agentes comunitários de saúde, quando presentes no domicílio em pelo menos uma das duas visitas realizadas.

Os dados foram coletados por estudantes de Nutrição veiculados ao projeto, sob coordenação e supervisão do professor pesquisador. Os procedimentos de coleta dos dados foram padronizados, por meio de capacitações, como medida de controle da qualidade e consistência das informações. Foi utilizado um questionário previamente padronizado, sendo coletadas informações sociodemográficas, avaliação antropométrica e da percepção corporal.

As medidas antropométricas coletadas foram: peso, altura e circunferência da cintura (CC), sendo utilizado uma balança digital portátil, estadiômetro portátil e fita métrica inelástica. Os indicadores analisados para classificação do estado nutricional antropométrico e distribuição de gordura corporal foram o índice de massa corporal (IMC $\left.=\mathrm{kg} / \mathrm{m}^{2}\right)$ e CC, respectivamente.

A classificação do IMC foi realizada conforme os critérios sugeridos pela World Health Organization $(\mathrm{WHO})^{7} \mathrm{e}$ Nutrition Screening Initiative (NSI) ${ }^{8}$, para adultos e idosos, respectivamente, enquanto para a distribuição de gordura corporal foi utilizada a recomendação da $\mathrm{WHO}^{7}$.

A imagem corporal foi verificada pela escala de nove silhuetas, proposta por Stunkard et al. ${ }^{9}$, com níveis desde magreza (silhueta 1) até a obesidade grave (silhueta 9), na qual o indivíduo escolhe o número da silhueta que considera mais semelhante à sua aparência corporal real (Percepção da Imagem Corporal Real - PICR) e também o número da silhueta que acredita ser a aparência corporal ideal para sua idade (Percepção da Imagem Corporal Ideal-PICl).

A avaliação da imagem corporal foi realizada por meio da subtração do valor de silhueta da PICR da silhueta da PICl (PICR - PICl). Para classificação do resultado, considerou-se que, quando a variação era igual a zero, o indivíduo era considerado satisfeito com a imagem corporal. Se diferente de zero, o indivíduo era considerado insatisfeito com a imagem corporal, de forma que, se a diferença fosse negativa, se classificou como insatisfação pela magreza e, se a diferença fosse positiva, era uma insatisfação pelo excesso de peso.

As análises estatísticas foram realizadas com o auxílio do software Statistical Package for the Social Science (SPSS), versão 20.0. No sentido de caracterizar a amostra estudada, as variáveis foram expressas por meio de valores absolutos e relativos, cálculo de tendência central (média) e de dispersão (desvio padrão). A normalidade dos dados foi verificada pelo teste de Shapiro-Wilk para todas as variáveis analisadas. Utilizou-se o teste qui-quadrado para verificar a associação entre o estado nutricional antropométrico e a percepção da 
imagem corporal (satisfeito e insatisfeito), adotando-se o nível de significância de $5 \%(p<0,05)$.

Este trabalho foi aprovado pelo Comitê de Ética e Pesquisa do Hospital Universitário de Aracaju/Universidade Federal de Sergipe (parecer $n^{\circ}$ 525.463). A participação no estudo ocorreu após todos os indivíduos serem esclarecidos acerca dos objetivos do trabalho e da garantia do seu anonimato e mediante assinatura do Termo de Consentimento Livre e Esclarecido.

\section{RESULTADOS}

Dentre os indivíduos avaliados, observou-se que a maioria era do sexo feminino $(87,6 \%)$ e de adultos $(67,4 \%)$. Em relação ao estado nutricional, a maioria apresentou excesso de peso $(61,8 \%)$ e $79,8 \%$ possuíam acúmulo de gordura abdominal (Tabela 1).

Conforme demonstra a Figura 1, a silhueta de percepção da imagem corporal ideal mais referida foi a $4(32,6 \%)$, enquanto as silhuetas de imagem corporal real mais mencionadas foram a $5(31,5 \%)$ e $7(20,2 \%)$. Ao analisar a satisfação com a imagem corporal, a maioria dos indivíduos demonstrou insatisfação pelo excesso de peso $(78,7 \%)$ e 6,7\% de insatisfação pela magreza (Figura 2).

Observou-se que houve associação entre estado nutricional antropométrico e percepção da imagem corporal $(p=0,013)$. Constatou-se que a maioria dos indivíduos com excesso de peso estava insatisfeita com a percepção corporal, assim como aqueles com adequado estado nutricional também apresentavam algum tipo insatisfação corporal (seja pela magreza ou pelo excesso de peso) (Tabela 2).
Tabela 1 - Caracterização dos usuários do HiperDia do município de Fátima-Bahia.

\begin{tabular}{lc}
\hline Variável & $\mathbf{n}(\%)$ \\
\hline Sexo & $11(12,4)$ \\
Masculino & $78(87,6)$ \\
\hline Feminino & \\
\hline Grupo etário & $60(67,4)$ \\
Adultos & $29(32,6)$ \\
Idosos & \\
\hline Estado nutricional & $1(1,1)$ \\
Magreza & $33(37,1)$ \\
Adequado & $55(61,8)$ \\
Excesso de peso & $18(20,2)$ \\
\hline Acúmulo de gordura abdominal & $71(79,8)$ \\
Adequado & Média (Mínimo - Máximo) \\
Excesso & $66,4(43,5-118,0)$ \\
\hline & $1,55(1,42-1,69)$ \\
Peso (kg) & $27,6(20,1-49,0)$ \\
Altura (m) & $92,7(73,0-138,5)$ \\
IMC (kg/m²) & \\
CC (cm) & \\
\hline CC = Circunferência da cintura; DP = Desvio padrão; IMC = Indice de massa corporal. &
\end{tabular}

$\overline{\mathrm{CC}}$ = Circunferência da cintura; $\mathrm{DP}$ = Desvio padrão; IMC = indice de massa corporal.

Tabela 2 - Associação entre estado nutricional antropométrico e percepção da imagem corporal de usuários do Programa HiperDia do município de Fátima-Bahia.

\begin{tabular}{lccc}
\hline \multirow{2}{*}{$\begin{array}{l}\text { Percepção } \\
\text { corporal }\end{array}$} & \multicolumn{3}{c}{$\begin{array}{c}\text { Classificação do estado nutricional } \\
\text { antropométrico }\end{array}$} \\
\cline { 2 - 3 } & Adequado & Excesso de peso & $\mathbf{p}$ \\
\cline { 2 - 3 } & $\mathbf{n}(\%)$ & $\mathbf{n}(\%)$ & \\
\hline Satisfeito & $9(26,5)$ & $4(7,3)$ & 0,013 \\
Insatisfeito & $25(73,5)$ & $51(92,7)$ & \\
\hline
\end{tabular}

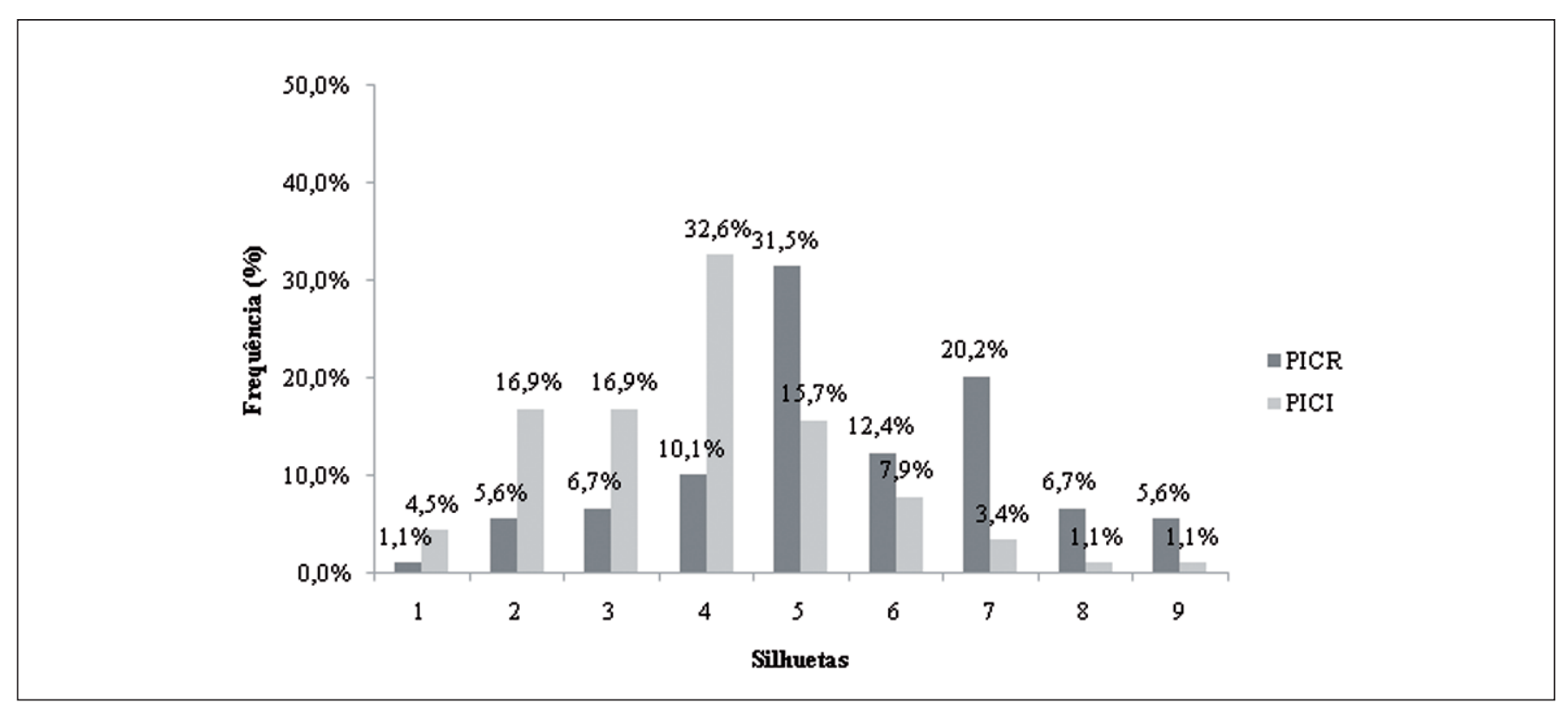

Figura 1 - Frequência de silhuetas de percepção corporal real e ideal de usuários do Programa HiperDia do município de Fátima-Bahia. PICI = Percepção da Imagem Corporal Ideal; PICR = Percepção da Imagem Corporal Real. 


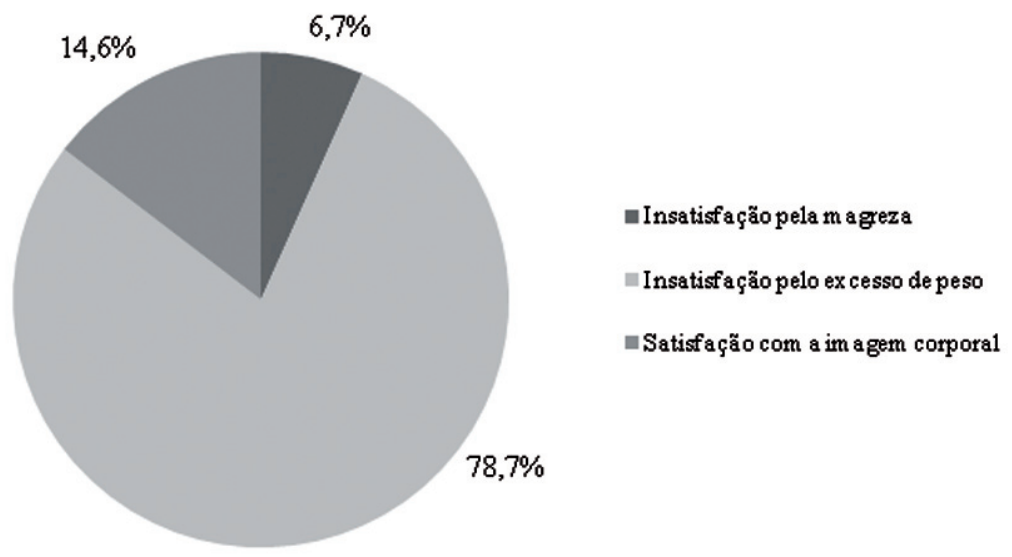

Figura 2 - Classificação da percepção da imagem corporal, conforme níveis de satisfação de usuários do Programa HiperDia do município de Fátima-Bahia.

\section{DISCUSSÃO}

No presente estudo, a maioria dos indivíduos apresentou insatisfação com a percepção corporal, sendo observada associação estatisticamente significante entre o estado nutricional e a percepção corporal. Os indivíduos avaliados possuíam uma visão de imagem corporal ideal baseada em um corpo que divergia do seu corpo real.

É importante destacar que há uma diferença entre estar dentro do padrão de beleza e estar com saúde. A estética não leva em consideração o bem-estar do indivíduo e, ao considerar que apenas a magreza é sinônimo de aceitação social e sucesso, gera uma incidência maior de transtornos mentais, principalmente ligados à autoimagem ${ }^{10}$. Este fato foi observado no presente estudo, uma vez que $73,5 \%$ dos indivíduos, mesmo com o estado nutricional adequado, acreditavam estar fora dos padrões de beleza.

No presente estudo, a silhueta de $\mathrm{PICl}$ mais referida foi a 4, enquanto as silhuetas de PICR mais mencionadas foram 5 e 7 , sendo que este resultado pode ter sido influenciado pela maioria da amostra ser do sexo feminino. Nos estudos de Damasceno et al. ${ }^{11}$ e Coelho \& Fagundes ${ }^{12}$, com mulheres adultas, assim como no estudo de Pereira et al. ${ }^{13}$ e Ferreira et al. ${ }^{14}$, conduzido com idosas, a $\mathrm{PICl}$ foi de 3 . Isso pode indicar que, possivelmente, o padrão de silhueta feminino se aproxima das características de um corpo mais magro e que o padrão ideal de silhueta feminina independe da faixa etária.

Além disso, ressalta-se que a insatisfação com a imagem corporal possui relação com o IMC excessivo, como observado no presente estudo, em que a maioria dos indivíduos com excesso de peso estava insatisfeita com o próprio corpo. Resultados semelhantes foram descritos na literatura em estudos realizados com adultos e idosos ${ }^{13-17}$.
Embora não tenha sido realizada análise por sexo no presente estudo, a literatura descreve que o sexo feminino está mais exposto à distorção da imagem corporal, independente da faixa etária ${ }^{18,19}$. Ferreira et al. ${ }^{19}$ constataram que a distorção da imagem corporal foi maior entre as mulheres e que, quanto maior o peso e o valor do IMC, maior foi o grau de insatisfação corporal. Ressalta-se que esse maior grau de insatisfação favorece ao maior desenvolvimento de autocrítica e um desejo pela aceitação social para se enquadrar no padrão de "corpo ideal". No entanto, para alcançar esse corpo desejável, há uma tendência para aumentar comportamentos de restrição alimentar e risco à saúde.

Diante disto, ao avaliar a percepção corporal, é preocupante a prevalência do distúrbio da insatisfação corporal, em que o corpo real não é considerado um corpo ideal. Isto parece estar refletindo uma idealização proposta por fatores midiáticos e sociais, no qual o corpo adequado está diretamente ligado à apresentação de silhuetas menores entre as mulheres e com destaque em massa magra para os homens ${ }^{20,21}$.

Constatou-se que o excesso de peso pode ser considerado um fator que interfere no grau de compreensão e formação da imagem corporal do indivíduo, assim como na sua satisfação corporal desta imagem formada, seja ela condizente ou não com a realidade. Ressalta-se que o ambiente externo, normas culturais e sociais influenciam diretamente na satisfação corporal dos indivíduos, seguindo as normas dos "padrões" atuais para magreza, o que pode contribuir para o desenvolvimento de distúrbios da imagem corporal, a depender da idade do indivíduo ${ }^{14}$.

Bosi et al. ${ }^{22}$ ressaltam que o culto ao corpo está ligado à imagem de poder, beleza e ascensão social e que, junto à insatisfação corporal, gera uma mobilização individual e até em massa para realização de atividade física exacerbada, 
cirurgias plásticas clinicamente desnecessárias e restrição alimentar, o que acarreta em agravos à saúde.

Além disso, é comum que os indivíduos que apresentam algum grau de distorção da imagem corporal exibam maior tendência a modificar seus hábitos alimentares, sem acompanhamento profissional, com o objetivo de alcançar seu peso desejado, independente desse peso ser o saudável ou não para sua estrutura física. Para tal, conceitos como "body image evaluation" e/ou "body image investment" são fundamentais para compreensão da satisfação corporal do indivíduo. Enquanto o primeiro diz respeito à satisfação/ insatisfação do indivíduo com o corpo, o segundo refere-se à importância cognitiva e comportamental que o indivíduo coloca na sua aparência física ${ }^{23}$. Dessa forma, sugere-se que o indivíduo que apresenta tendência a modificar seus hábitos alimentares ou tenta perder peso, tanto pode ser insatisfeito com seu corpo como pode dar muita importância a sua autoavaliação ${ }^{23,24}$.

Vale ressaltar que o método de escala de nove silhuetas, proposto por Stunkard et al. ${ }^{9}$, possui como vantagem boa validade na estimativa do estado nutricional, além de ser o instrumento mais popular para ser adaptado e utilizado em adultos $^{25}$. Porém, essa escala não foi construída com base em dados antropométricos medidos, o que pode ser um viés, justificando também as adaptações que sofre para sua aplicabilidade em estudos.

\section{CONCLUSÃO}

A maioria dos indivíduos apresentava excesso de peso e insatisfação com a imagem corporal. Além disso, mesmo aqueles indivíduos com o estado nutricional adequado expressaram insatisfação corporal. Essa insatisfação pode causar problemas nutricionais e psicológicos, mesmo em adultos e idosos, isso porque, em busca da aceitação social por meio do enquadramento do corpo nos padrões impostos pela sociedade na qual estão inseridos, os indivíduos podem desenvolver transtornos alimentares, acarretando problemas psicológicos e biológicos. Portanto, é imprescindível uma avaliação da imagem corporal dos indivíduos por parte dos profissionais da saúde, auxiliando a prática clínica e garantindo uma atenção integral ao indivíduo. É interessante estabelecer parcerias entre nutrição e psicologia, seguindo princípios do Sistema Único de Saúde, que trazem a universalidade, equidade e integralidade para auxílio da desconstrução do pensamento generalizado que a sociedade impõe sobre o corpo.

\section{AGRADECIMENTOS}

Agradecemos aos usuários do Programa HiperDia, pela cooperação e participação; à Maria Edvânia Alves Santos, membro do Núcleo de Apoio à Saúde da Família do município de Fátima-Bahia, pelo suporte logístico.

\section{REFERENCIAS}

1. Gondim MR, Cunha SFS, Souza SG, Schmidt A, Barros DD. Percepção da imagem corporal de idosas praticantes de um programa de hidroginástica. EFDeportes.com. 2011;15(153). [cited 2019 Oct 23]. Available from: https:// www.efdeportes.com/efd153/imagem-corporal-de-idosasde-hidroginastica.htm

2. Maciel FT, Ferreira JS. Percepção da auto-imagem corporal em relação ao estado nutricional de escolares do ensino médio em Campo Grande, MS. EFDeportes.com. 2010 [cited 2019 Oct 23]. Available from: http://www.efdeportes.com/efd146/autoimagem-corporal-em-relacao-ao-estado-nutricional.htm

3. Tribess S, Virtuoso Junior JS, Petroski EL. Estado nutricional e percepção da imagem corporal de mulheres idosas residentes no nordeste do Brasil. Ciênc Saúde Coletiva. 2010;15(1):31-8.

4. Silva PGE. Estado nutricional e imagem corporal dos idosos cadastrados na estratégia de saúde da família em Campina Grande-PB [Trabalho de Conclusão de Curso - Graduação em Fisioterapia]. Campina Grande: Universidade Estadual da Paraíba; 2011.

5. Leal SA. Estado de saúde auto-percebido, índice de massa corporal e percepção da imagem corporal em utentes dos cuidados de saúde primários. [Dissertação de Mestrado]. Lisboa: Universidade de Lisboa; 2009.

6. Zenith AR, Marques CRC, Dias JC, Rodrigues RCC. Avaliação da percepção e satisfação da imagem corporal em usuários do programa academia da cidade em Belo Horizonte - Minas Gerais. Revista e-Scientia. 2012;5(1):9-17.

7. World Health Organization. Obesity: preventing and managing the global epidemic. Report of a WHO Consultation Group. Genebra: World Health Organization; 2000. [cited 2019 Oct 23]. Available from: http://whqlibdoc.who.int/trs/WHO_TRS_894.pdf

8. The Nutrition Screening Initiative. Incorporating nutrition screening and interventions into medical practice: a monograph for physicians. Washington: American Academy of Family Physicians. The American Dietetic Association. National Council on Aging Inc; 1994.

9. Stunkard AJ, Sorenson T, Schlusinger F. Use of the Danish Adoption Register for the study of obesity and thinness. In: Kety SS, Rowland LP, Sidman RL, Matthysse SW, eds. The genetics of neurological and psychiatric disorders. New York: Raven Press; 1983. p.115-20.

10. Pinto DCD, Quadrado RP. Imagens em construção: satisfação corporal e transtornos alimentares em acadêmicos da área da saúde. RELACult. 2018;4(759):1-11.

11. Damasceno VO, Lima JRP, Vianna JM, Vianna VRA, Novaes JS. Ideal physical type and body image satisfaction of regular walkers. Rev Bras Med Esporte. 2005;11(3):181-6.

12. Coelho EJN, Fagundes TF. Imagem corporal de mulheres de diferentes classes econômicas. Motriz Rev Educ Fis. 2007;13(2 supl):S37-43.

13. Pereira EF, Teixeira CS, BorgattoAF, Daronco LSE. Relação entre diferentes indicadores antropométricos e a percepção da imagem corporal em idosas ativas. Rev Psiq Clín. 2009;36(2):54-9.

14. Ferreira AA, Menezes MFG, Tavares EL, Nunes NC, Souza FP, Albuquerque NAF, et al. Estado nutricional e autopercepção da imagem corporal de idosas de uma Universidade Aberta da Terceira Idade. Rev Bras Geriatr Gerontol. 2014;17(2):289-301.

15. Saur AM, Pasian SR. Satisfação com a imagem corporal em adultos de diferentes pesos corporais. Aval Psicol. 2008;7(2):199-209. 
16. Chaim J, Izzo H, Sera CTN. Cuidar em saúde: satisfação com imagem corporal e autoestima de idosos. O Mundo da Saúde. 2009;33(2):175-81.

17. Fonseca CC, Chaves ECL, Pereira SS, Barp M, Moreira AM, Nogueira DA. Autoestima e satisfação corporal em idosas praticantes e não praticantes de atividades corporais. Rev Educ Fís/ UEM. 2014;25(3):429-39.

18. Molina MJR, Izquierdo DG, Pérez MLV, Moreno RL, Vallejo EN, Toral MV, et al. Imagem corporal y satisfacción corporal en adultos: diferencias por sexo y edad. Rev Iberoamericana de Psicologia del Ejercicio y el Deporte. 2015;10(1):63-8.

19. Ferreira PDAA, Sampaio RMM, Cavalcante ACM, Monteiro TF, Pinto FJM, Arruda SPM. Caracterização do comportamento alimentar e estado nutricional de adultos. Motricidade. 2018;14(1):252-8.

20. Barroso DR, Almeida LIR, Kulnig AM. Mídia e construção da imagem corporal em adolescentes do gênero feminino. Rev Bras Reabilitação e Atividade Física. 2012;1(1):53-62.
21. Monteiro GN, Silva SR, Mazzardo T, Araújo ND, Aburachid LMC. Nível de (in)satisfação corporal de mulheres praticantes de treinamento em circuito. Pensar a Prática. 2018;21(1):41-52.

22. Bosi MLM, Luiz RR, Morgado CMC, Costa MLS, Carvalho RJ. Autopercepção da imagem corporal entre estudantes de nutrição: um estudo no município do Rio de Janeiro. J Bras Psiquiatr. 2006;55(2):108-13.

23. Cash TF, Smolak L. Body image: a handbook of science, practice, and prevention. $2^{\text {nd }}$ ed. New York: Guilford Press; 2011.

24. Fredrickson BL, Roberts TA, Noll SM, Quinn DM, Twenge JM. That swimsuit becomes you: sex differences in self-objectification, restrained eating, and math performance. J Pers Soc Psychol. 1998;75(1):269-84.

25. Moraes C, Anjos LA, Marinho SMSA. Construção, adaptação e validação de escalas de silhuetas para autoavaliação do estado nutricional: uma revisão sistemática da literatura. Cad Saúde Pública. 2012;28(1):7-19.

\section{Local de realização do estudo: Universidade Federal de Sergipe, Departamento de Nutrição, Lagarto, SE, Brasil.}

Conflito de interesse: Os autores declaram não haver. 